\title{
Presynaptic Voltage-Dependent Calcium Channels and Regulation of the Transmitter Release in the Peripheral Nervous System
}

\author{
E. A. Bukharaeva ${ }^{a, b}$, A. N. Tsentsevitsky ${ }^{a}$, A. L. Vasin ${ }^{a}$, D. V. Samigullin ${ }^{a}$, and E. E. Nikolsky ${ }^{a, b}$ \\ ${ }^{a}$ Kazan Institute of Biochemistry and Biophysics, Kazan Scientifc Center, Russian Academy of Sciences, \\ Kazan; ellyab@mail.ru \\ ${ }^{b}$ Kazan State Medical University, Kazan, Russia \\ DOI: $10.1134 / \mathrm{S} 1990747809030143$
}

Calcium ions entering into the nerve terminal through voltage-dependent calcium channels during action potential initiate neurotransmitter release and thereby play an important role in the transmission of excitation. In addition to the amount of released neurotransmitter quanta (the quantal content of the endplate potential), another important characteristic of exocytosis is the kinetics of release of individual quanta that form total multiquantal response. Previously, we showed the dependence of the temporal parameters of neurotransmitter quantum release on the extracellular calcium ion concentration, but it remained unclear which calcium channel types are involved in the modulation of the quantal content and release kinetics. It is commonly accepted that, in contrast to the central nervous system, wherein the secretion take part various types of voltage-dependent calcium channels, exocytosis in the peripheral nervous system of adult animals mainly occurs owing to one type of calcium channels. This is the P/Q type ( $\mathrm{Ca}_{V} 2.1$ in the latest classification) blocked with FTX toxin and $\omega$-agatoxin IVA in mammalian neuromuscular junction. In frog synapses, this is the $\mathrm{N}$ type channels $\left(\mathrm{Ca}_{\mathrm{V}} 2.2\right)$, which are blocked by the $\omega$-conotoxin GVIA. At the same time, the ability of FTX, a specifc P/Q channel blocker, to decrease the quantal content in frog synapses and effects of dihydropyridines that block $\mathrm{L}$ type $\left(\mathrm{Ca}_{\mathrm{V}} 1.2\right)$ channels in the developing Xenopus synapses, and $\mathrm{L}$ type $\left(\mathrm{Ca}_{\mathrm{V}} 1.2\right)$ channels activator effects in frog synapses, taken together, indicate the possibility of participation of other types of calcium channels in the exocytosis regulation in peripheral synapses. The possible role of changes in the activity of not only P/Q channels, but also other calcium channel types, in modulation of the temporal parameters of neurotransmitter release was shown in peripheral synapses of mouse and crayfish. In order to identify the type of voltage-dependent calcium channels modulating of the quanta secretion in frog and mouse synapses, the effects of specific blockers of various channel types on the endplate potentials quantal content and transmitter release kinetics were analyzed. On isolated Rana ridibunda neuromuscular preparation of $\mathrm{m}$. cutaneus pectoris and on the mouse diaphragm the evoked uniquantal endplate currents were registered by the extracellular electrodes and the fluctuations of the real synaptic latency were estimated. Blocking of the P/Q-, N-, and L-channels in nerve-muscle junction of the frog by the appropriate specific blockers led to a decrease in the amount of released quanta, as well as in the synaptic latency variance, that indicate the synchronization of the process of synaptic vesicles exocytosis. Obtained results indicate that in low-calcium solution the kinetics of quanta secretion in the frog synapse may be regulated by $\mathrm{N}-, \mathrm{P} / \mathrm{Q}-$, and L-calcium channels. In the mouse nerve muscle junctions at low calcium concentration and infrequent stimulation, when asynchrony of the secretion was more pronounced, the block of the P/Q-channels led to a decrease in the amount of released quanta without changing the kinetics of their secretion, while blocking the L-type channels only decreased variance of synaptic latency. The fact that the number of released quanta and the time course of their secretion may change independently when various types of voltage-dependent calcium channels are blocked witnesses that the role of N-, P/Q-, and L-channels in modulation of the process of synaptic vesicles exocytosis is different.

This study was supported by the Russian Foundation for Basic Research (project no. 08-04-00923) and the United States Civilian Research and Development Foundation (project no. RUB1-2823-KA06). 